Background and aims Congenital malformations (CM) are defined as abnormal structure of the organism resulting from disrupted embryogenesis. Many factors influence the appearance of CM. Regarding different criteria and authors, the incidence of $\mathrm{CM}$ at newborns is between $2-7 \%$. The aim of this paper was to determine the incidence of $\mathrm{CM}$ at liveborns delivered at SHGO "Mother Teresa" - Cair, Skopje, during the five years period (2000-2004) and five years period (2005-2009). Also, the distribution among organ system had been analysed.

Methods A retrospective analysis of 19097 liveborns delivered at SHGO Cair, during 2000-2004, has been performed. Database (Access 2000) from Neonatal Unit has been used. The incidence and percentage of CM among different systems have been determined and a retrospective analysis of 15293 liveborns delivered at SHGO Cair, during 2005-2009, has been performed. Database (Access 2005) from Neonatal Unit has been used. The incidence and percentage of CM among different systems have been determined.

Results Period 2000-2004, among a total of 19097 liveborns, 736 or $3,85 \%$ have had CM. the incidence between different years was:4,28\% in 2000, 3,92\%-2001, 3,79\%- 2002, 3,20\%2003 and 3,98\% in 2004. Regarding different organ systems the distibution was: $58,1 \%$ of all CM were the anomalies of musceulosceletal system, 14,6\% - cardiopathies, 8,1\% - anomalies of uro-genital system 7,2\% -CM of gastro -intestinal system, etc. Perid 2005-2009, among a total of 15293 liveborns, 573 or $3,75 \%$ have had CM. the incidence between different years was: $4,02 \%$ in 2005, 3,42\%-2006, 3,22\%- 2007, 4,23\%-2008 and $3,85 \%$ in 2009 . Regarding different organ systems the distibution was: $40,31 \%$ of all CM were the anomalies of musceulosceletal system, 21,81\% - cardiopathies, 20,07\% - anomalies of uro-genital system, 12,04\% -CM of gastro -intestinal system, $5,76 \%$ of CNS, etc.

Conclusions During the five years period (2000-2004), the incidence of CM is 3,85\%, which is in accordance with data from literature. Among years, the incidence varies from 3,2 to $4,3 \%$. During the five years period 2005-2009, the incidence of $\mathrm{CM}$ is $3,75 \%$, which is in accordance with data from literature. Among years, the incidence varies from 3,2 to $4,3 \%$. The anomalies of musculoskeletal system are the most frequent, followed by those from cardiovascular, uro-genital, gastrointestinal and central nervous system. Comparing the five-year periods 2000-2004 and 2005-2009 shows almost identical incidence. Congenital malformations still remain an important medical and social problem requesting more serious nationwide engagement, as in medical aspect, socioeconomic, ecological, etc.

\section{P0-0692 THE VALUE OF RISK FACTORS IN SCREENING FOR DEVELOPMENTAL DYSPLASIA OF THE HIP (DDH). CAN SELECTIVE SCREENING REPLACE UNIVERSAL SCREENING?}

B Kurdi. Pediatric, Hamad General Hospital, Doha, Qatar

\subsection{6/archdischild-2014-307384.1331}

Background Ultrasonography is accepted as a reliable tool in detecting DDH. Universal screening is criticised to be financially demanding, time consuming and leads to overtreatment. Selective ultrasonography is proposed as the alternative.

Methods A retrospective study of all newborn in a period of 2 years. All newborn with risk for $\mathrm{DDH}$ or positive clinical signs were screened with ultrasonography (selective screening). Data of babies who presented after the age of 6 months with DDH.

Results Between May 2008 and April 2010, 33768 live births were registered. 966 newborn had risk for DDH, 532 with breech presentation, 81 had positive family history, 440 were premature, 191 product of multiple pregnancy, 67 had foot abnormality, 3 with torticollis, and 7 with other anomalies.

12 babies who were born in the same period and were not screened because they had neither risk for DDH or positive clinical finding presented after the age of 6 months with dislocated hips, In screened group 15 newborn could be discovered early with DDH, whereas 12 were missed and presented late.

Conclusions Only 56\% of newborn with DDH could be detected with the selective screening program, which is statistically not acceptable, and selective screening program in our hand is not an adequate tool to detect DDH. Risk for DDH can detect only 1 out 9 with DDH only, and cannot be considered alone as tool for screening.

\section{PO-0693 UNBOUND BILIRUBIN CORRELATES WITH SKIN BILIRUBIN MEASURED IN JAUNDICED NEONATES}

E Letamendia-Richard, R Benammar, S Oucherif, M Baron, L Giorgi, D De Luca. Division of Pediatrics and Neonatal Critical Care, APHP-South Paris University Hospitals, Paris, France

\subsection{6/archdischild-2014-307384.1332}

Background and aims The American Academy of Paediatrics recommends the hour-specific evaluation of either skin (SB) or total serum bilirubin as screening tool for neonatal jaundice. However, serum bilirubin is not a good predictor of kernicterus, while SB represents a molecular specie already extravasated and passed into a tissue. Circulating unbound bilirubin (UB) is actually related to the neurotoxicity, being the portion of bilirubin capable to freely pass from circulation to the tissues. We aimed at exploring the relationship between SB and UB.

Methods Simultaneous measurements of SB (using 2nd generation transcutaneous devices) and UB were performed in 35 term jaundiced neonates.

Results In term neonates (mean GA: 38 weeks; BW 3095 g; postnatal age 74,8 h; TSB $250.8 \mu \mathrm{mol} / \mathrm{L}$ (SD 63); UB 0.48 (SD $0.2) \mu \mathrm{g} / \mathrm{dL}$ ), a positive correlation was found between $\mathrm{SB}$ and UB $(r=0.70 ; \mathrm{p}<0.001 ;$ Figure 1$)$. This correlation remained significant after adjustment for birth weight, gestational or postnatal age (partial correlation $\mathrm{r}=0.68, \mathrm{p}<0.001 ; \mathrm{r}=0.69$, $\mathrm{p}<0.001 ; \mathrm{r}=0.64, \mathrm{p}<0.001)$.

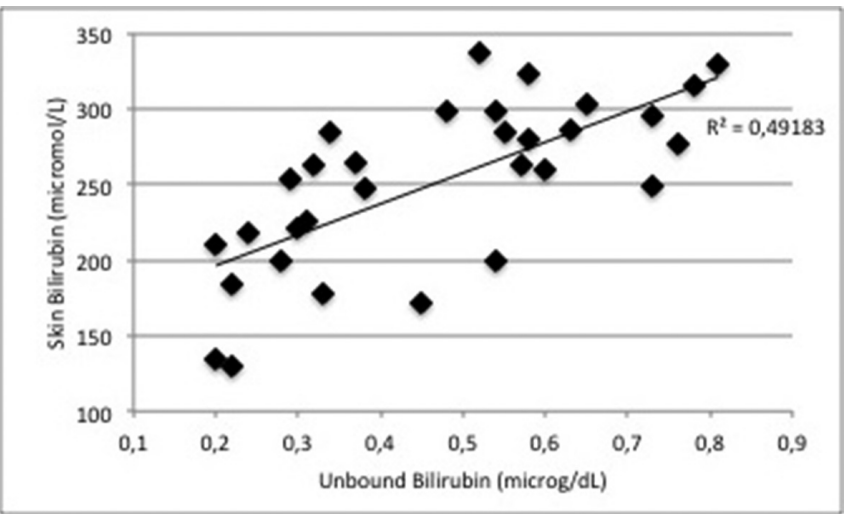

Abstract P0-0693 Figure 1 


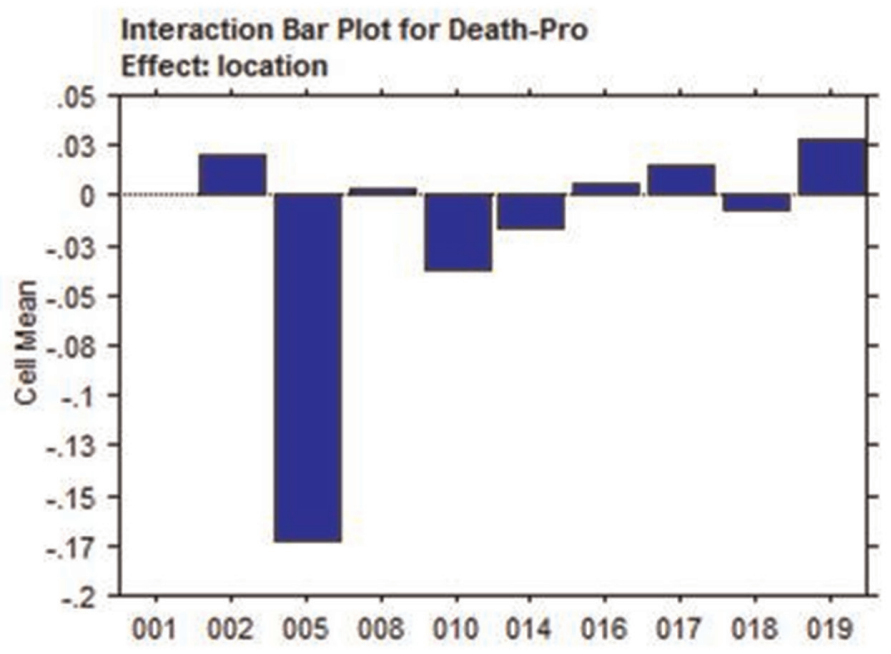

\section{Abstract P0-0694 Figure 1}

Conclusions These preliminary data suggest that SB is strongly correlated to UB. SB could be related to neurotoxicity as it may be formed by UB passed from circulation to a tissue. Further investigations are needed to clarify this relationship and possible influencing factors.

\section{PO-0694 RISK-ADJUSTED MORTALITY OF VLBW IN TAIWAN- A POPULATION-BASED STUDY}

${ }^{1} \mathrm{YJ}$ Lin, ${ }^{1} \mathrm{YC}$ Lin, ${ }^{1} \mathrm{CH}$ Lin, ${ }^{1} \mathrm{CC}$ Huang, ${ }^{2} \mathrm{KI}$ Tsou, ${ }^{3}$ Taiwan Premature Infant Developmental Collaborative Study Group. 'Pediatrics, National Cheng Kung University and Hospital, Tainan, Taiwan; ${ }^{2}$ Pediatrics, Fu Jen Catholic University, New Taipei, Taiwan; ${ }^{3}$ Taiwan PBF, Premature Baby Foundation of Taiwan, Taipei, Taiwan

\subsection{6/archdischild-2014-307384.1333}

Background and aims Preterm babies have higher mortality than terms. Risk-adjusted mortality (RAM) is useful for making comparisons among different NICUs. GA, BBW, sex, singleton birth and antenatal steroid have been used to estimate mortality (M) of preterm. The aims of this study are 1 . To compare the performance of GA, BW, and Logistic Regression (LR) in predicting $\mathrm{M}$ of VLBW infants. 2. To compare the RAM in different areas and periods.

Methods Cohort data from 2000 to 2011 were used. M is defined as death prior to discharge. Exclusion criteria included 1) Transferred after $24 \mathrm{~h}$ of age; 2) Death within $24 \mathrm{~h}$ of admission and 3) Lethal malformation. We developed a LR model to predict $\mathrm{M}$ [expected probability (Pro)]. ROC curves were used for assessing performance of predicting M. To compare the RAM, we calculated (O-E) Pro (observed Pro expected Pro) values in each patient and used these values for comparisons.

Results 9207 VLBWs were enrolled. The calculated probability of death by LR model was: $\mathrm{P}=1 /\left(1+\mathrm{e}^{-\mathrm{Z}}\right)$, where $\mathrm{e}=$ natural logarithms and $\mathrm{z}=(-0.62 \cdot[$ prenatal steroid] $)-(0.219 \bullet \mathrm{GA})-$ $(0.004 \cdot \mathrm{BBW})-(0.327 \cdot[$ singleton $])+(0.286 \bullet[\mathrm{male}])+8.438$. Area under ROC were 0.858 for LR (95\% CI: $0.847-0.869$ ), 0.841 for BBW (95\% CI: $0.829-0.853)$ and 0.827 for GA $(95 \%$ CI: $0.815-0.839)$.

There were significant differences of RAM in different locations and years (Figure 1).

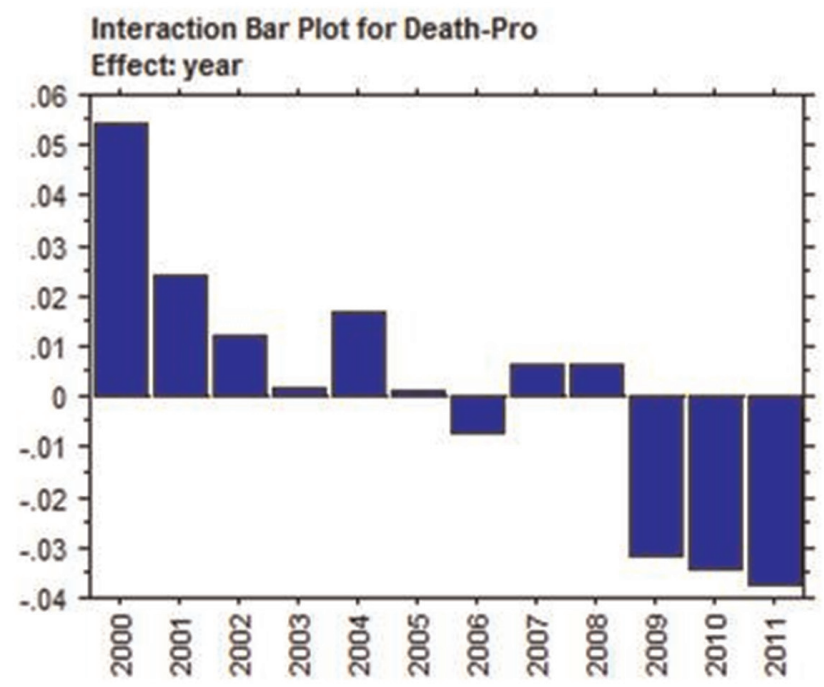

Conclusions The use of LR is better than GA and BW in predicting $\mathrm{M}$ of VLBWs. RAM can be used as a tool for quality improvement.

\section{P0-0695 DELIVERY ROOM MANAGEMENT OF EXTREMELY PRETERM INFANTS IN A TERTIARY NEONATAL CENTRE: A TEN YEAR REVIEW}

L McGrory, COF Kamlin, AR Rafferty, LS Owen, JA Dawson, PG Davis. Department of Newborn Research, The Royal Women's Hospital, Parkville Melbourne, Australia

\subsection{6/archdischild-2014-307384.1334}

Background and aims In the delivery room (DR), respiratory support for preterm infants has traditionally been provided through mechanical ventilation following intubation, which is known to increase neonatal morbidities including BPD and sepsis. The Nasal CPAP or Intubation at birth for very preterm infants (COIN) Trial demonstrated that infants spontaneously breathing at birth may be managed in the DR with non invasive support (CPAP) rather than intubation, which may be preferred. We aimed to review the management of extremely preterm infants in the DR at the Royal Women's Hospital (RWH) during a ten-year period and evaluate whether respiratory support practices differed during and after the COIN trial.

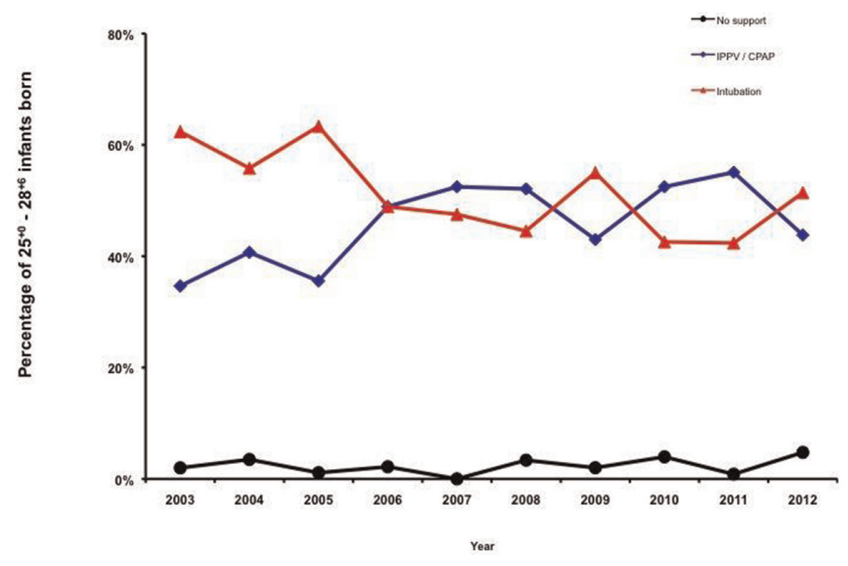

Abstract P0-0695 Figure 1 